www.jmscr.igmpublication.org

Impact Factor 5.84

Index Copernicus Value: 71.58

ISSN (e)-2347-176x ISSN (p) 2455-0450

crossref DOI: _https://dx.doi.org/10.18535/jmscr/v5i10.191

Journal Of Medical Science And Clinical Research

IGM Publication

An Official Publication of IGM Publication

\title{
Comparative Evaluation of Spectral Domain Optical Coherence Tomography versus Fundus Fluorescein Angiography for the detection of Diabetic Macular Edema
}

\author{
Authors \\ Prof. Veshal Madan', Dr Renu Magdum², Dr Somnath Ghosh ${ }^{1}$ \\ ${ }^{1}$ Dr DY Patil Institute of Optometry \& Visual Sciences, Pimpri Pune \\ ${ }^{2}$ Dr D Y Patil Medical College and Hospital, Pimpri, Pune \\ Corresponding Author \\ Prof. Veshal Madan
}

Dr DY Patil institute of Optometry \& Visual Sciences, Dr D Y Patil Vidyapeeth (Deemed University)

Sant Tukaram Nagar, Pimpri, Pune - 411018,

Phone: 020-27805952, Email: director.optom@dpu.edu.in

\begin{abstract}
Background: Drastic incidence of DME in largest populated nation in world has direct effect on VTDR which is the major cause of irreversible blindness in DR subjects. Therefore, the need for reliable, safe and real time detection of DME is an utmost need for detection and prevention of the disease. Hence, the purpose of the study was to compare the efficacy of two well known diagnostic tools, SD-OCT \& FFA for the detection of DME.

Methods: A prospective study was carried out on 308 eyes of 154 type II DM patients to compare the detection potential between two popular Retinal Diagnostic Techniques, SD-OCT and FFA for DME. The parameters such as focal macular edema, diffuse macular edema and mixed edema were evaluated with FFA. Afterwards, IR edema, sub retinal fluid and mixed edema were diagnosed with SD-OCT for the same pool of patients. Clinical features in DME using reference 'EDTRS' scale of measurement.

Results: Noninvasively 305 (99.1\%) eyes were detected positive for various type of DME by SD-OCT similarly 282 (91.6) eyes were detected positive by FFA. The prevalence of different types of DME such as cystoids, diffuse, focal and mixed was 7.8\%, 13\%, 7.1\% and $63.7 \%$ as diagnosed with FFA. Whereas, SRF, IR edema and mixed was $4.9 \%, 3.6 \%$ and $90.6 \%$ as diagnosed with SD-OCT. DME was not detected by FFA $8.4 \%$ of overall study population. Whereas, DME was not detected $0.9 \%$ eyes by SD-OCT. Overall performance was better in detection of DME with SD-OCT compared to FFA.

Conclusion: The present study showed the efficacy of SD-OCT over FFA in Reliable and safe detection of DME in Type II Diabetic Mellitus patients were significantly more by SD-OCT and was easy and comparable to diagnostic findings of FFA specifically in cases of sub retinal macular as well choroidal edema due DME by $S D-O C T$.

Keywords: Diabetic Macular Edema, Spectral Domain-Optical Coherence Topography, Fundus Fluorescein Angiography.
\end{abstract}




\section{Introduction}

Diabetes Mellitus (DM) is a group of metabolic diseases characterized by hyperglycaemia due to malfunction secretion or absorption of insulin or both which results in irreversible damage to eyes, kidney, brain, heart with blood vessels \& nerves of the body. ${ }^{[1]}$ Diabetes mellitus (DM) is a major cause of avoidable blindness in both the developing and the developed countries. ${ }^{[2,3]}$

Diabetic retinopathy, one of the earliest frequent and serious complications of diabetes, remains a major public health problem with significant socioeconomic implications, affecting approximately $50 \%$ of diabetic subjects and remaining the leading cause of blindness in working-age populations of industrialized countries ${ }^{[4]}$. Patients with diabetic retinopathy (DR) are 25 times more likely to become blind than non-diabetics. Drastic incidence of DME in largest populated nation in world has direct effect on major cause of irreversible blindness in DR subjects. Therefore, the need for reliable, safe and real time detection of DME is an utmost need for detection and prevention of the disease. The purpose of the study was to compare effectiveness of Spectral Domain-Optical Coherence Tomography (SDOCT) over Fundus Fluorescein Angiography (FFA) for the detection of Diabetic Macular Edema (DME).

\section{Methods}

Three hundred and eight eyes of 154 patients with Diabetic Macular Edema (DME) were recruited for the present study from the ophthalmology department in a tertiary hospital in western Maharashtra for the period of 2 years. Type II Diabetes Mellitus with reduced visual acuity due to DME were enrolled in study. Patients with surgical intervention on retina and vitreous pathology were excluded from the study. All the patients were underwent detail ophthalmological examination including refraction, slit lamp biomicroscopy, ophthalmoscopy. The initial diagnosis of DME was done by using +90 diopter (D) lens through slit lamp biomicroscopy.
Afterwards, all the DME diagnosed patients were underwent fundus photography (FP) for the entire quadrant by Zeiss Fundus Camera, Fundus Fluorescein Angiography (FFA) and Spectral Domain Optical Coherent Topography (SD-OCT). Ophthalmological investigations were done by an ophthalmologist with the presence of a general physician. DME was classified based on the EDTRS classification. ${ }^{[5]}$

The parameters such as focal macular edema, diffuse macular edema and mixed edema were evaluated with FFA. Afterwards, IR edema, sub retinal fluid and mixed edema were diagnosed with SD-OCT for the same pool of patients. Clinical features in DME using reference 'EDTRS' scale of measurement. Sub classification by presence of Sub Retinal Fluid and Intra Retinal Edema were the hallmark findings of SD-OCT while detection of areas of non- perfusion \& hyper perfusion due subsequent Neovascularisation in case of Ischemic macular edema were only detected by FFA. Ethics approval was obtain from institutional review board prior to the study. Descriptive statistical analysis was used for the present study.

\section{Results}

Three hundred and eight eyes of 154 patients with Diabetic Macular Edema (DME) were analysed in the present study. The age of the patient ranged from 40 years to 76 years with the average of 60.64 years. The table clearly shows that maximum number of patients $(50.6 \%)$ was from the age group 61-70 years followed by 51-60 years $(27.3 \%)$. Nearly 89 percent patients were above 50 years. It has also found that out of 154 patients about $70.13 \%$ were male and rest 29.87 $\%$ was females. Thus the group was dominated by elderly males. 


\section{JMSCR Vol||05||Issue||10||Page 29624-29630||October}

Figure 1: Number of eyes in each age group

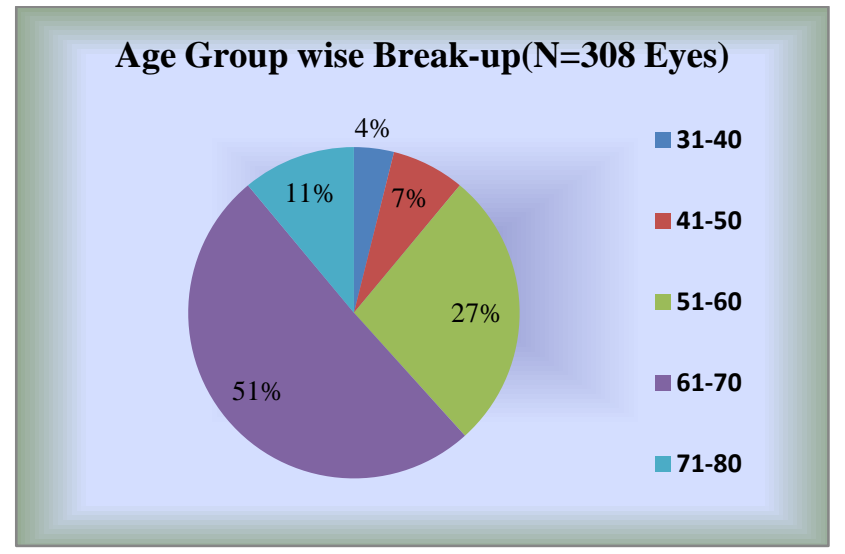

Past history of all patients shows that the complaint of reduced vision was noticed by them only for the last 2 to 4 years. The average duration was noted at 2.53 years. It was also noted that all patients were suffering from Type II DM. The average duration of DM was reported as 13.07 years. The detailed descriptive statistics is given below.

Figure 2: Age Group versus DM

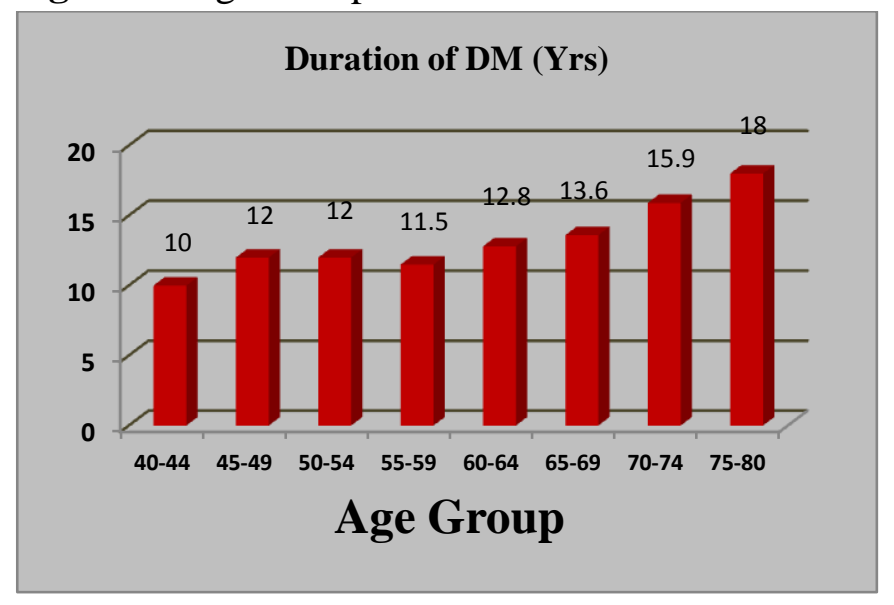

It is seen that major portion of eyes consisting of $48.7 \%$ are from $6 / 24$ category, followed by the $6 / 18$ which contribute to $35.4 \%$. Remaining eyes in various grades were having individual share less than $10 \%$ of total eyes examined.
Figure 3: Break-up of Uncorrected Vision for the DME patients

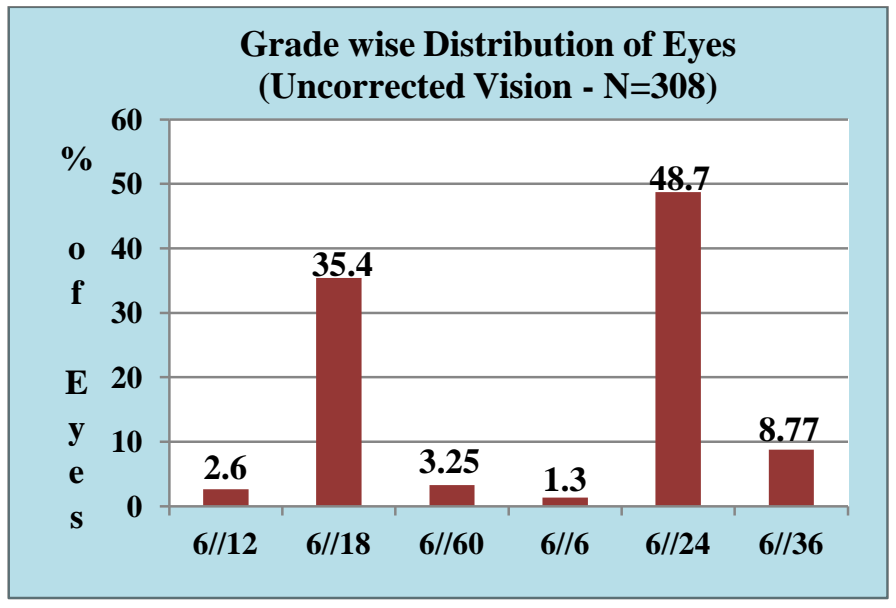

After refraction the vision was improved by one line. It is seen that major portion of eyes consisting of $46.8 \%$ are from 6/18 category, followed by the $6 / 12$ which contributes to $33.1 \%$ and $6 / 24$ which contributes $15.3 \%$. Remaining eyes in various grades were having individual share less than $10 \%$ of total eyes examined.

Table 4: Break-up of corrected Vision for the DME patients

\begin{tabular}{|l|c|c|}
\hline Grade of Vision & No. of Eyes & Percent to Total \\
\hline $6 / 12$ & 102 & 33.1 \\
\hline $6 / 18$ & 144 & 46.8 \\
\hline $6 / 6$ & 4 & 1.3 \\
\hline $6 / 9$ & 6 & 1.9 \\
\hline $6 / 24$ & 47 & 15.3 \\
\hline $6 / 36$ & 5 & 1.6 \\
\hline Total & 308 & 100.0 \\
\hline
\end{tabular}

Various procedures were carried out for examining eyes for detection of DME by various methods. All eyes of all patients were firstly examined with Slit Lamp Biomicroscopy as the primary method. Out 308 eyes, 83.1\% were detected as DME by Slit Lamp Biomicroscope. The results are as shown below table.

Table 5: Detection of DME through Slit Lamp Biomicroscopy

\begin{tabular}{|l|c|c|}
\hline & No. of Eyes & Percent to total \\
\hline $\mathbf{N}$ & 52 & 16.9 \\
\hline $\mathrm{Y}$ & 256 & 83.1 \\
\hline Total & 308 & 100.0 \\
\hline
\end{tabular}




\section{JMSCR Vol||05||Issue||10||Page 29624-29630||October}

Out 308 eyes, $96.8 \%$ were detected as DME by Fundus Photography which is comparatively better to primary investigation technique. Under this procedure only $3.2 \%$ eyes were not detected to have DME. The results are as shown below table.

Table 6: Detection of DME through Fundus Photography

\begin{tabular}{|l|c|c|}
\hline & No. of Eyes & Percent to total \\
\hline $\mathbf{N}$ & 10 & 3.2 \\
\hline $\mathrm{Y}$ & 298 & 96.8 \\
\hline Total & 308 & 100.0 \\
\hline
\end{tabular}

Fundus Fluorescein Angiography (FFA): Using this procedure, abnormalities due to DME such as various types of leakages due to focal, diffuse and cystoids were attempted to be detected.

FFA Focal: Using this procedure out of 308 eyes 110 eyes $(35.7 \%)$ were not detected for DME, but could detect balance 198 eyes $(64.3 \%)$.

Table 7: Detection of FFA Focal through Fundus Fluorescein Angiography

\begin{tabular}{|l|l|l|}
\hline & No. of Eyes & Percent to Total \\
\hline $\mathbf{N}$ & 110 & 35.7 \\
\hline $\mathrm{Y}$ & 198 & 64.3 \\
\hline Total & 308 & 100.0 \\
\hline
\end{tabular}

FFA Diffuse: Using FFA procedure, out of 308 eyes, $76.6 \%$ eyes were not found for DME diffuse abnormality. It could detect only $23.4 \%$ eyes as affected by DME diffuse.

Table 8: Detection of FFA Diffuse through Fundus Fluorescein Angiography

\begin{tabular}{||l|c|c|}
\hline & No. of Eyes & Percent to Total \\
\hline$N$ & 236 & 76.6 \\
\hline Total & 72 & 23.4 \\
\hline
\end{tabular}

FFA Cystoids: Cystoids Spaces were noted by FFA procedure for $68.2 \%$ eyes - a little over $2 / 3^{\text {rd }}$. Out of 308 eyes 98 were not detected to have cystoids. Table below shows the details.
Table 9: Detection of FFA Cystoids through Fundus Fluorescein Angiography

\begin{tabular}{|l|c|c|}
\hline & No. of Eyes & $\begin{array}{c}\text { Percent to } \\
\text { Total }\end{array}$ \\
\hline $\mathbf{N}$ & 98 & 31.8 \\
\hline Y & 210 & 68.2 \\
\hline Total & 308 & 100.0 \\
\hline
\end{tabular}

Results of detection of various abnormalities by FFA procedure are consolidated in the following figure.

Figure 4: Detection of various abnormalities of DME by FFA procedure

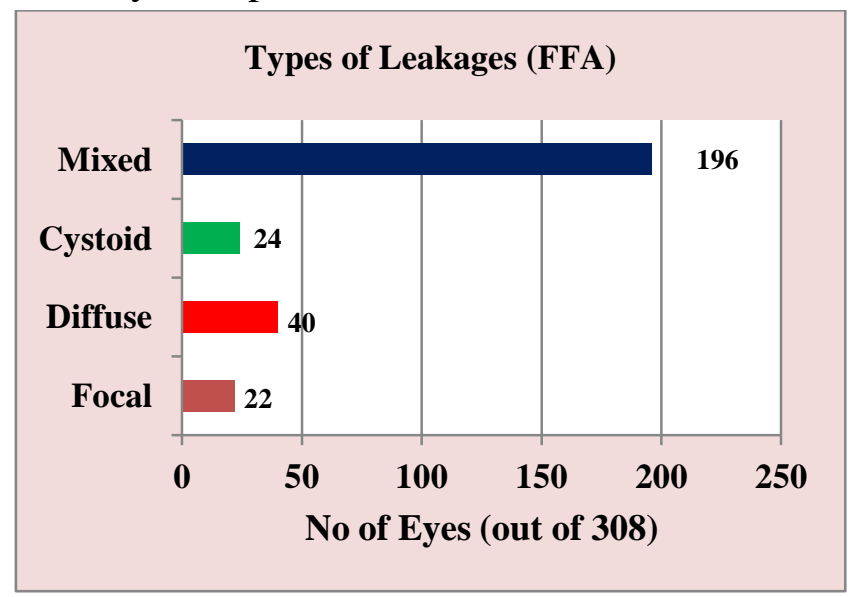

\section{Spectral Domain-Optical Coherence}

Tomography (SD-OCT): It's a comparatively advanced procedure for examining eyes than FFA for detecting various other defects associated with DME. The detailed outputs for various abnormalities are elaborated in table below.

Inter Retinal Edema: It is seen that a little less than $2 / 3^{\text {rd }}$ eyes were detected to have IR edema by SD -OCT.

Table 10: Inter Retinal Edema detected by SD OCT.

\begin{tabular}{|l|c|c|}
\hline & No. of Eyes & $\begin{array}{c}\text { Percent to } \\
\text { Total }\end{array}$ \\
\hline $\mathbf{N}$ & 108 & 35.1 \\
\hline$Y$ & 200 & 64.9 \\
\hline Total & 308 & 100.0 \\
\hline
\end{tabular}

Combined Signs: Over $60 \%$ eyes were detected for absence of combined signs. 
Table 11: Combined Signs detected by SD -OCT

\begin{tabular}{|l|c|c|}
\hline & No. of Eyes & $\begin{array}{c}\text { Percent to } \\
\text { Total }\end{array}$ \\
\hline $\mathbf{N}$ & 187 & 60.7 \\
\hline $\mathbf{Y}$ & 121 & 39.3 \\
\hline Total & 308 & 100.0 \\
\hline
\end{tabular}

Cystoids Spaces: Cystoids to the tune of $73 \%$ were successfully detected by SD -OCT procedure.

Table 12: Cystoids Spaces detected by SD -OCT.

\begin{tabular}{||l|c|c|}
\hline & No. of Eyes & $\begin{array}{c}\text { Percent to } \\
\text { Total }\end{array}$ \\
\hline $\mathbf{N}$ & 84 & 27.3 \\
\hline $\mathrm{Y}$ & 224 & 72.7 \\
\hline Total & 308 & 100.0 \\
\hline
\end{tabular}

Sub Retinal Fluid: Presence of Sub Retinal Fluid was found in $76.9 \%$ eyes by SD-OCT procedure

Table 13: Sub Retinal Fluid detected by SD OCT.

\begin{tabular}{|l|c|c|}
\hline & No. of Eyes & $\begin{array}{c}\text { Percent to } \\
\text { Total }\end{array}$ \\
\hline $\mathrm{N}$ & 71 & 23.1 \\
\hline $\mathrm{Y}$ & 237 & 76.9 \\
\hline Total & 308 & 100.0 \\
\hline
\end{tabular}

Results of detection of various abnormalities by SD-OCT procedure are consolidated in the following figure.

Figure 5: Detection of various abnormalities of DME by SD-OCT procedure

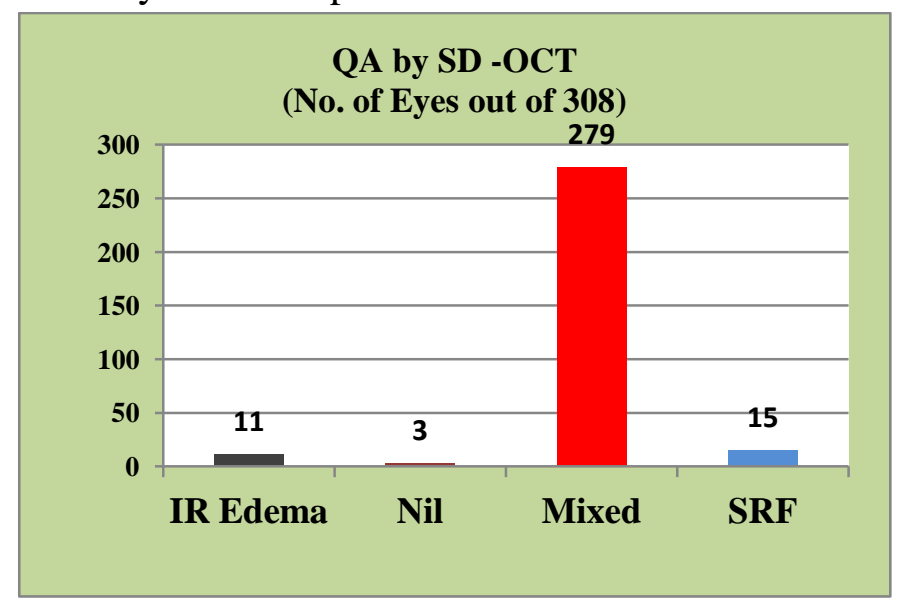

Results from figure 4 and figure 5 when compared together, it shows that there are two common categories viz. Nil and Combined / Mixed. When the figures of number of eyes are compared, it shows that SD-OCT is more powerful or efficient to detect abnormalities than FFA. FFA could detect 26 eyes with no abnormalities however SDOCT detected only 3 eyes. This also shows that there were 23 eyes with some kind of abnormality which FFA failed to detect but SD-OCT did it correctly. Same is the case with mixed / combined category. This shows the efficiency of SD- OCT procedure over FFA in Clinical testing of eyes.

\section{Discussion}

The prevalence of Diabetic Macular edema (DME) increases with age as $50.6 \%$ were in $61-70$ years age group which correlate to previous studies. ${ }^{[6,7,8]}$ In the present study, presence of Intraretinal Edema and Sub Retinal Fluid were directly related to visual acuity reduction detected by SD- OCT is a major advantage over FFA.

The present study was carried out to determine the potential diagnostic tool between FFA and SDOCT. The study showed promising results by both FFA and SD-OCT. Based on Optical Coherence Tomography, 3 types of Diabetic Macular Oedema, Intraretinal edema, Sub Retinal Fluid and mixed macular edema were detected. In the present study, Intraretinal edema (11), Sub Retinal Fluid (15), and 279 eyes showed combined pattern. So most common pattern in OCT was combined pattern. On Optical Coherence Tomography, Cystoid Macular Oedema (CME) was seen in $72.7 \%$ eyes while on Fundus Fluorescein Angiography $68.2 \%$ eyes showed CME which is demonstrating importance of OCT in early detection of foveal involvement which is not seen on FFA.

Sub Retinal Fluid $76.9 \%$ on OCT not seen on FFA, which is explains severe loss of vision in diabetic macular oedema. So FFA with severe diffuse leak masks CME and serous detachments. Measurement of CFT was possible in OCT which is important cause of centre involving Macular 
oedema not possible to diagnose by FFA. FA is known to be a sensitive method for qualitative assessment of fluid leakage in diabetic macular edema; FA is an invasive procedure, with side effects ranging from nausea to its rare complication of anaphylaxis and death.

OCT is non-invasive, comfortable, safe, and fast and can be repeated as often as is required and offers an alternative to FA in the follow-up of changes in retinal thickness after laser photocoagulation and intravitreal steroid injections. FA is still essential for the assessment of the foveal perfusion state which cannot be demonstrated by OCT. After an initial FA, OCT seems to be a useful non-invasive tool in the close follow-up of the effectiveness of treatment modalities in diabetic maculopathy.

\section{Conclusion}

There is need for safe and effective patient friendly method for detection of pathophysiology effects on Diabetic Mellitus Patients with visual function resulting in irreversible vision loss.

The active role of eye care practitioner including optometrist is detect early and refer to an retinal specialist for management as there are 62.6 million persons who are suffering from Diabetic related complication among the affected persons due to VTDR(Visual threatening DR). Therefore need to Compare two modalities SD-OCT versus FFA \& their ability early detect Macular edema due to DM is a significant step in ensuring appropriate technique is used in an eye care for early detection \& referral for DME.

\section{References:}

1. Saikat Sen, Raja Chakraborty, Biplab De. Complications of Diabetes Mellitus. Diabetes Mellitus in 21st Century, pp 69100.

2. Ramandeep Singh, Kim Ramasamy, Chandran Abraham, Vishali Gupta, and Amod Gupta. Diabetic retinopathy: An update. Indian J Ophthalmol. 2008 MayJun; 56(3): 179-188.
3. Saad Hajar, MD, Ali Al Hazmi, MD, Mustafa Wasli, MD, Ahmed Mousa, PhD, and Mansour Rabiu. Prevalence and causes of blindness and diabetic retinopathy in Southern Saudi Arabia. Saudi Med J. 2015; 36(4): 449-455.

4. B Askira, B Mubi. Blindness In Patients With Diabetes Mellitus Attending A Diabetes Clinic At The University Of Maiduguri Teaching Hospital, Maiduguri. The Internet Journal of Ophthalmology and Visual Science. 2012 Volume 9 Number 1.

5. Sapna Gangaputra, James F. Lovato, Larry Hubbard, Matthew D Davis,1 Barbara A Esser,1 Walter T. Ambrosius,2 Emily Y. Chew,3 Craig Greven,4 Letitia H Perdue,2 Wai T. Wong, ${ }^{*}, 3$ Audree Condren,3 Charles P. Wilkinson,5 Elvira Agrón,3 Sharon Adler,6 Ronald P Danis, 1 and ACCORD Eye Research Group. Comparison of Standardized Clinical Classification with Fundus Photograph Grading for the assessment of Diabetic Retinopathy and Diabetic Macular Edema Severity. Retina. 2013 Jul-Aug; 33(7).

6. Ryan Lee, Tien Y. Wong and Charumathi Sabanayagam. Epidemiology of diabetic retinopathy, diabetic macular edema and related vision loss. Eye and Vision20152:17

7. Rohit Varma, MD, 1 Neil M. Bressler, MD,2 Quan V. Doan, PharmD,3 Michelle Gleeson, PhD,3 Mark Danese, PhD,3 Julie K. Bower, $\mathrm{PhD}, 4$ Elizabeth Selvin, $\mathrm{PhD}, 2$ Chantal Dolan, PhD,5 Jennifer Fine, PhD,5 Shoshana Colman, $\mathrm{PhD}, 5$ and Adam Turpcu, PhD5. Prevalence of and Risk Factors for Diabetic Macular Edema in the United States. JAMA Ophthalmol. 2014 Nov; 132(11): 1334-1340.

8. Diabetic macular edema. Bandello $\mathrm{F}$, attaglia Parodi M Lanzetta P Loewenstein A Massin P, Menchini F, Veritti D. Diabetic macular edema (DME) is the 
largest cause of visual acuity loss in diabetes

9. (Chapter 1 ; Diabetic Macular Edema; Conceic ; ao Lobo, Isabel Pires, and Jos`eCunha-Vaz;bookreveiw)

10. Kaveeshwar SA, Cornwall J. The current state of diabetes mellitus in India. The Australasian Medical Journal. 2014;7(1): 45-48. doi:10.4066/AMJ.2013.1979. 